Louisiana State University

LSU Digital Commons

Faculty Publications

Department of Biological Sciences

$1-1-2015$

\title{
Effect of 12 weeks of periodized resistance training upon total plasma adiponectin concentration in healthy young men
}

Greggory R. Davis

University of Louisiana at Lafayette

Jacqueline M. Stephens

Pennington Biomedical Research Center

Arnold G. Nelson

Louisiana State University

Follow this and additional works at: https://digitalcommons.Isu.edu/biosci_pubs

\section{Recommended Citation}

Davis, G., Stephens, J., \& Nelson, A. (2015). Effect of 12 weeks of periodized resistance training upon total plasma adiponectin concentration in healthy young men. Journal of Strength and Conditioning Research, 29 (11), 3097-3104. https://doi.org/10.1519/JSC.0000000000000894

This Article is brought to you for free and open access by the Department of Biological Sciences at LSU Digital Commons. It has been accepted for inclusion in Faculty Publications by an authorized administrator of LSU Digital Commons. For more information, please contact ir@lsu.edu. 


\title{
Effect of 12 Weeks of Periodized Resistance Training Upon Total Plasma Adiponectin Concentration in Healthy Young Men
}

\author{
Greggory R. Davis, ${ }^{1}$ Jacqueline M. Stephens, ${ }^{2,3}$ and Arnold G. Nelson ${ }^{4}$ \\ ${ }^{1}$ School of Kinesiology, University of Louisiana at Lafayette, Lafayette, Louisiana; ${ }^{2}$ Pennington Biomedical Research Center, \\ Baton Rouge, Louisiana; ${ }^{3}$ Department of Biological Sciences, Louisiana State University, Baton Rouge, Louisiana; and ${ }^{4}$ School \\ of Kinesiology, Louisiana State University, Baton Rouge, Louisiana
}

\begin{abstract}
Davis, GR, Stephens, JM, and Nelson, AG. Effect of 12 weeks of periodized resistance training upon total plasma adiponectin concentration in healthy young men. J Strength Cond Res 29(11): 3097-3104, 2015-The effects of resistance training on adiponectin have thus far yielded equivocal results. However, the effect of periodized resistance training on plasma adiponectin in offspring of type 2 diabetic and nondiabetic (ND) parents (first-degree family history) has yet to be determined. Untrained healthy young men with and without a first-degree family history of type 2 diabetes were assigned to an exercise intervention group (E) or an active control group (C). The $\mathrm{E}$ group performed a 12week periodized resistance training program, whereas the C group did not participate in any structured exercise program. Fasting plasma samples were obtained pre- and posttraining. Total plasma adiponectin changed significantly in the $E$ group $(33.7 \pm 14.7 \%, p=0.025)$ but not in the $C$ group (10.8 $\pm 19.2 \%)$. Offspring of type 2 diabetic parents subjects had significantly greater improvements in plasma adiponectin (69.3 $\pm 34.8 \%$ ) compared with ND subjects $(-3.2 \pm 29.9 \%, p=0.046)$. Regular aerobic exercise was significantly correlated with average plasma adiponectin ( $r=$ 0.32), whereas first-degree family history of type 2 diabetes $(r=-0.58)$ and decreases in body fat percentage $(r=$ -0.77) were inversely correlated with average plasma adiponectin. Periodized high-intensity resistance training seems to increase plasma adiponectin, particularly in individuals with a first-degree family history of type 2 diabetes or those who experience a significant loss of fat mass. The direct correlation between regular aerobic exercise and adiponectin further suggests that a combination of aerobic and
\end{abstract}

Address correspondence to Greggory R. Davis, grd4805@louisiana.edu. 29(11)/3097-3104

Journal of Strength and Conditioning Research

(c) 2015 National Strength and Conditioning Association resistance training yields greater improvements in plasma adiponectin compared with resistance training alone.

KEY WoRDS first-degree family history, type 2 diabetes, western blot

\section{INTRODUCTION}

$\Omega$ he offspring of type 2 diabetic (OD) parents are often referred to as individuals with a first-degree family history of diabetes. These individuals are at a greater risk for developing type 2 diabetes compared with the offspring of nondiabetic (ND) parents because of a greater genetic predisposition to the disease (3). Not only is it important for OD to identify early signs of insulin resistance to prevent the progression of the disease, but it is also important for these individuals to find mechanisms to actively enhance insulin sensitivity. One biomarker that has been shown to be directly suppressed in OD is the circulating levels of the adipocyte-derived hormone, adiponectin, which plays an important role in insulin sensitivity and glucose and lipid metabolism $(9,19)$. In addition to its role in the regulation of glucose and lipid metabolism, adiponectin has anti-inflammatory properties (34). Chronic low-grade inflammation, which is associated with obesity, diabetes, and cardiovascular disease, is also associated with low levels of circulating adiponectin, as reviewed by Ouchi and Walsh (32). Thus, adiponectin has been deemed as an antiinflammatory adipokine (34) and has shown to play an important role in the suppression of inflammation and is associated with enhanced insulin sensitivity. Adiponectin levels are generally elevated among healthy lean populations and suppressed among obese, insulin-resistant, or type 2 diabetic populations (9). Although lifestyle modifications, specifically exercise intervention studies, have irrefutably demonstrated numerous benefits in the prevention of type 2 diabetes via enhanced glucose uptake and insulin sensitivity $(6,11,16)$, plasma adiponectin responses to exercise are much less clear, particularly in OD populations.

Long-term exercise training has been shown to reduce inflammation (15), improve insulin sensitivity (29), and 
TABLE 1. Comparison of baseline characteristics for the $E$ and $C$ groups. *

\begin{tabular}{llcc}
\hline Subject characteristics & $\mathrm{C}(n=12)$ & $\mathrm{E}(n=12)$ & $p$ \\
\hline Age (y) & $22.1 \pm 1.0$ & $20 \pm 0.3$ & 0.074152 \\
Weight $(\mathrm{kg})$ & $85.8 \pm 1.1$ & $82.5 \pm 2.8$ & 0.506933 \\
BMl & $28.1 \pm 1.2$ & $25.9 \pm 1.0$ & 0.178958 \\
Percent body fat & $23.8 \pm 6.0$ & $19.3 \pm 1.3$ & 0.052728 \\
Family history T2D (\%) & $16.7 \pm 11.2$ & $38.5 \pm 14.0$ & 0.238284 \\
Reg. aerobic ex. (\%) & $33.3 \pm 14.2$ & $23.1 \pm 12.2$ & 0.588996 \\
Reg. resistance ex. (\%) & $41.7 \pm 14.9$ & $15.4 \pm 10.4$ & 0.16309 \\
Reg. caffeine (\%) & $38.6 \pm 8.5$ & $53.8 \pm 15.4$ & 0.29461 \\
\hline
\end{tabular}

${ }^{*} \mathrm{C}=$ active control group; $\mathrm{E}=$ exercise group; $\mathrm{BMI}=$ body mass index; Family history T2D $(\%)=$ percentage of participants who reported mother or father with type 2 diabetes; Reg. aerobic ex. $(\%)=$ percentage of participants who reported regular aerobic exercise; Reg. resistance ex. $(\%)=$ percentage of participants who reported regular resistance exercise; Reg. caffeine $(\%)=$ percentage of participants who reported regular caffeine consumption. Values are presented as mean $\pm S D$.

responses to a periodized resistance training program. Periodized resistance training programs have been shown to elicit superior gains in neuromuscular, (28) metabolic, anti-inflammatory $(4,33)$, and endocrine $(23,24)$ adaptations compared with nonperiodized programs. Therefore, it is possible that periodized resistance training programs may induce changes in plasma adiponectin that have not been observed in previous studies, which in large part, have implemented nonperiodized resistance training programs. Furthermore, several studies have examined exercise responses in aging and type 2

improve blood lipid (25) and glucose profiles (7). Therefore, exercise should theoretically be an effective method for increasing plasma adiponectin levels. Thus far, however, highly variable responses have been observed in studies examining exercise-induced changes in plasma adiponectin. Several studies have demonstrated that plasma adiponectin tends to increase after chronic high-intensity exercise training $(13,21)$ or in the presence of weight loss $(26,37)$, whereas other studies show no change in plasma adiponectin after an exercise intervention despite enhanced insulin action $(17,29)$. Up to this point, no studies to our knowledge have examined

TABLE 2. Week-to-week periodization. *

\begin{tabular}{cccc}
\hline Week & Sets & Repetitions & $\begin{array}{c}\text { Intensity } \\
(\% 1 R M)\end{array}$ \\
\hline 1 & $\begin{array}{c}\text { Familiarization \& } \\
\text { baseline testing }\end{array}$ & & \\
2 & 3 & 10 & 60 \\
3 & 3 & 6 & 70 \\
4 & 6 & 10 & 60 \\
5 & 3 & 4 & 80 \\
6 & 3 & 8 & 60 \\
7 & 4 & 6 & 70 \\
8 & 4 & 2 & 85 \\
9 & 5 & 5 & 80 \\
10 & 3 & 12 & 55 \\
11 & 4 & 4 & 85 \\
12 & 3 & 6 & 70 \\
13 & 5 & 4 & 85 \\
14 & Repeat baseline & & \\
\multicolumn{4}{c}{ testing } \\
\hline
\end{tabular}

$* \% 1 \mathrm{RM}=$ percentage of 1 repetition maximum. diabetic populations $(5,27)$, but few have examined young nondiabetic individuals. Of those studies, it is unknown whether the parents of the young healthy subjects had type 2 diabetes. By examining healthy young individuals with and without type 2 diabetic parents, it may be possible to determine whether genetic variation affects circulating levels of adiponectin after an exercise training intervention.

The purpose of the current study was to determine if a 12week periodized high-intensity resistance training program would elicit changes in circulating plasma adiponectin levels in healthy young men with and without a first-degree family history of type 2 diabetes. If periodized resistance training is effective in improving plasma adiponectin levels in the absence of weight loss, particularly in OD men, practitioners should consider using this type of training program, even for nonathlete populations, as opposed to a nonlinear training cycle for individuals with a first-degree family history of type 2 diabetes. We hypothesized that adiponectin levels would increase after the exercise intervention and that greater changes would be observed in the OD compared with the ND.

\section{Methods}

\section{Experimental Approach to the Problem}

Currently enrolled students at the university were recruited to participate in the study and were randomly assigned to the exercise intervention (E) or active control (C) groups. The E group participated in a 12 -week exercise intervention, whereas the $\mathrm{C}$ group maintained their normal exercise habits but did not engage in any structured exercise program for the duration of the study. An additional 1 week of familiarization and baseline testing was included at the onset of the study and a 1-week follow-up for retesting was also included at the conclusion of the study for both groups; thus, the total duration of the study was 14 weeks. To minimize subject variability and minimize extraneous variables, 


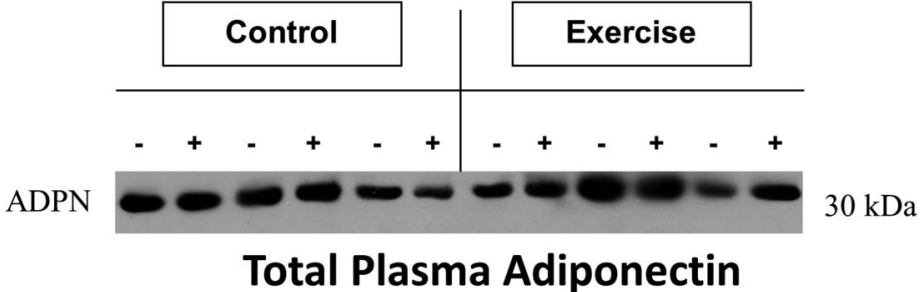

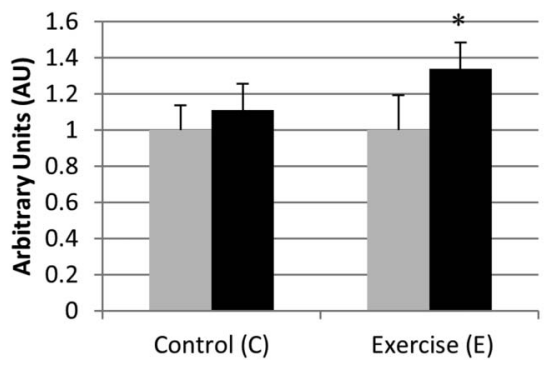

Figure 1. Exercise intervention modestly increases circulating levels of total adiponectin. Bar graphs represent relative optical densities of total adiponectin (ADPN) normalized to the respective pre-exercise control band intensities derived from the same gel. * $p \leq 0.05$ compared with pre-exercise; $-=$ pre-exercise; $+=$ postexercise; $n$ $=12$ per group. Values are presented as mean $\pm S D$. subjects were asked to abstain from alcohol and caffeine consumption and strenuous physical activity for 48 hours before baseline testing. All procedures were repeated after the conclusion of the exercise intervention to determine changes in plasma adiponectin and changes in anthropometric measures.

\section{Subjects}

Untrained healthy men $(n=24)$ who were a minimum of 18 years of age, range 18-33 years (average $\pm S D)(21.8 \pm 3.8)$ with a body mass index $<30$ were recruited to participate in the study. The E group consisted of 4 OD subjects and $8 \mathrm{ND}$ subjects. The $\mathrm{C}$ group consisted of $2 \mathrm{OD}$ subjects and $10 \mathrm{ND}$

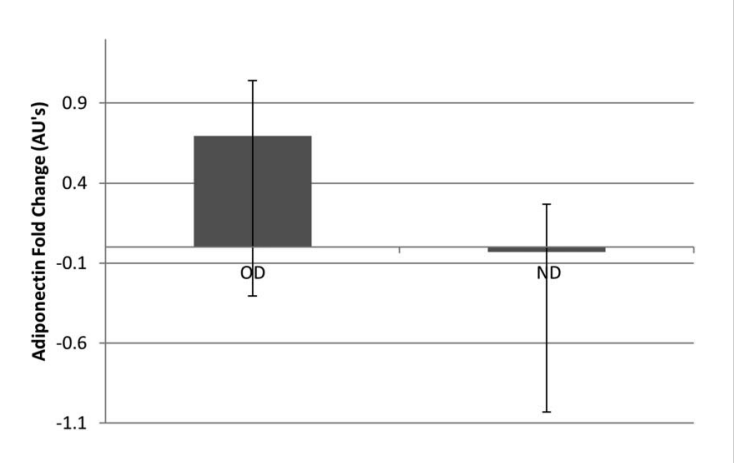

Figure 2. Changes in total adiponectin in diabetic offspring (OD) vs. nondiabetic offspring (ND). Fold change in plasma adiponectin pre- to postexercise in OD $(n=4)$ vs. ND $(n=8)$ in the E group. ${ }^{*} p \leq 0.05$ compared with ND. Values are presented as mean $\pm S D$. puncture to determine initial plasma adiponectin values. Blood was collected into sodium heparin vacutainers (Becton, Dickinson and Company, Franklin Lakes, NJ, USA); plasma was then separated and stored at $-80^{\circ} \mathrm{C}$ until further analysis. Height and weight were measured using a Detecto medical scale (Cardinal Scale Manufacturing Co., Webb City, MO, USA), body fat was determined via the 7-site skinfold technique using Lange skinfold calipers (Beta Technology, Inc., Cambridge, MD, USA) and included the chest, triceps, abdomen, thigh, suprailiac, subscapular, and midaxillary sites, per the American College of Sports Medicine (ACSM) guidelines (1), and the International Physical Activity Questionnaire was used to determine exercise habits. First-degree family history of type 2 diabetes was determined by asking each subject if his mother or father had been diagnosed with type 2 diabetes. Caffeine consumption was determined by asking each subject how many cups of coffee, tea, soda, energy drinks, and energy shots were typically consumed in a week. Regular caffeine consumption was considered a combination of any of the following equating to $\geq 5$ per week: 8 ounce cup of coffee or tea, 12 ounce caffeinated soda, or 1 energy shots or drinks. Regular aerobic exercise was considered $\geq 150$ minutes of total aerobic exercise per week, as per the ACSM guidelines. All baseline measurements and testing procedures were repeated at the conclusion of the exercise intervention after 48 hours of abstinence from alcohol consumption, caffeine consumption, and strenuous physical activity.

\section{Exercise Protocol}

As outlined in Table 2, after a familiarization session, the initial strength of each subject was determined by 1 
TABLE 3. Comparison of fold change in plasma adiponectin pre- to postintervention. *

\begin{tabular}{lcccc}
\hline Group & NDC & NDE & ODC & ODE \\
\hline Average fold change & $0.35 \pm 0.57$ & $0.03 \pm 0.3$ & $-0.47 \pm 0.07$ & $0.69 \pm 0.35 \dagger$ \\
\hline \multirow{2}{*}{$N D C=$ nondiabetic control group $(n=10) ;$ NDE $=$ nondiabetic exercise group $(n=9) ;$} \\
ODC $=$ diabetic offspring control group $(n=2) ;$ ODE $=$ diabetic offspring exercise group $(n=$ \\
3). Values are presented as mean $\pm S D$. \\
$\dagger p \leq 0.05$ compared with NDE.
\end{tabular}

pull-downs, and push press. All exercises were completed with barbell free weights with the exception of glut ham raises, which were done with weight plates, pull-ups, which were done with body weight, and lat pull-downs, which were done on weight-stacked machine. To minimize the risk of overtraining and injury, no single muscle group was targeted on consecutive exercise sessions. repetition maximum (1RM) for free-weight squat, freeweight bench press, and free-weight deadlift. These values were then used to determine relative exercise intensity for these individual exercises. All strength measurements were repeated upon completion of the 12 -week exercise intervention during the retesting week. A week-by-week description for the exercise intervention is described in Table 2. Resistance training was performed 3 times per week for $40-50$ minutes per exercise session, and each session was supervised by the research personnel. Each exercise session was separated by a minimum of 48 hours. Subjects were required to participate in a minimum of $80 \%$ of the exercise sessions to be included in the analysis.

Total work volume varied from week to week, and each day consisted of 3-5 exercises depending on the number of sets and repetitions. Three exercises were used for high-volume days, whereas 4 or 5 exercises were used for low-volume days. For example, Monday of week 2 consisted of 3 sets of 10 repetitions for back squats, Romanian deadlifts, and good mornings at a lower intensity (3 exercises total), whereas Wednesday of week 5 consisted of 3 sets of 4 repetitions for flat bench press, incline bench press, push press, pull-ups, and bent-over rows at a higher intensity ( 5 exercises total). Specific exercises varied from week to week and included back squats, front squats, flat bench press, incline bench press, conventional deadlifts, Romanian deadlifts, sumo deadlifts, bent-over rows, upright rows, good mornings, glut ham raises, pull-ups, lat

\section{Plasma Analysis}

All plasma samples were analyzed to determine the level of total plasma adiponectin via Western blot. Protein was quantified for each sample as determined by bicinchoninic acid assay (Sigma-Aldrich, St. Louis, MO, USA), and equal amounts of protein from each sample were subjected to electrophoresis. Samples were separated in $7.5 \%$ polyacrylamide (acrylamide from National Diagnostics) gels containing sodium dodecyl sulfate and transferred to a nitrocellulose membrane in $25 \mathrm{mM}$ Tris, $192 \mathrm{mM}$ glycine, and $20 \%$ methanol. After the transfer, the nitrocellulose membrane was blocked overnight in $4 \%$ milk at $4^{\circ} \mathrm{C}$ and then probed with human-anti-adiponectin (Thermo Scientific MA1-054) using a $1: 1000$ dilution in $1 \%$ bovine serum albumin in $1 \times$ Tris-buffered saline tween. Results were visualized with horseradish peroxidase-conjugated mouse-antihuman secondary antibody (1:1000 in $1 \%$ nonfat milk in $1 \times$ Trisbuffered saline tween) and enhanced chemiluminescence. Densitometric analysis of blots was performed using ImageJ software, and figures were produced using Prism 6 Graphpad software (Graphpad Software, Inc., La Jolla, CA, USA).

\section{Statistical Analyses}

Baseline subject characteristics between $\mathrm{E}$ and $\mathrm{C}$ groups were analyzed using an unpaired $t$-test. Paired $t$-tests were used to determine changes in strength and plasma adiponectin pre- to postintervention within groups. A 2-way analysis of variance was used to compare fold changes in adiponectin in the $\mathrm{C}$ and $\mathrm{E}$ groups and $\mathrm{OD}$ and ND. Associations between variables were investigated using Pearson's correlation analyses, and significant differences were identified using $\mathrm{Tu}-$ key post hoc tests. Multiple linear regression analysis was used to determine the degree of contribution of first-degree family history, changes in body weight, and changes in body fat percentage to explain plasma adiponectin levels. An 
TABLE 5. Adiponectin correlation. *

\begin{tabular}{lcr}
\hline Characteristic & $\begin{array}{c}\text { Pearson } \\
\text { correlation }\end{array}$ & \multicolumn{1}{c}{$p$} \\
\hline Change in body weight & -0.75 & $<\mathbf{0 . 0 1}$ \\
Change in BMl & -0.35 & 0.07 \\
Change in body fat (\%) & -0.77 & $<\mathbf{0 . 0 1}$ \\
Family history T2D (\%) & -0.58 & $<\mathbf{0 . 0 1}$ \\
Reg. aerobic ex. (\%) & 0.32 & $\mathbf{0 . 0 2}$ \\
Reg. resistance ex. (\%) & 0.21 & 0.19 \\
Reg. caffeine (\%) & 0.25 & 0.11 \\
\hline
\end{tabular}

${ }^{*} \mathrm{BMI}=$ body mass index; Family history $\mathrm{T} 2 \mathrm{D}(\%)=$ percentage of participants who reported mother or father with type 2 diabetes; Reg. aerobic ex. $(\%)=$ percentage of participants who reported regular aerobic exercise; Reg. resistance ex. $(\%)=$ percentage of participants who reported regular resistance exercise; Reg. caffeine $(\%)=$ percentage of participants who reported regular caffeine consumption. Bold indicates statistically significant correlations.

alpha level of 0.05 was used to determine statistical significance. Power or effect size was determined for statistically significant results. All values are presented as mean $\pm S D$. All statistical analyses were completed using SAS version 9.2 (SAS Institute, Inc., Cary, NC, USA).

\section{Results}

The exercise intervention had minor, but significant, effects on plasma adiponectin concentration $\left(p=0.025, \eta^{2}=0.03\right)$. The average fold change in plasma adiponectin expression in E group was $0.34 \pm 0.15$ compared with baseline values,

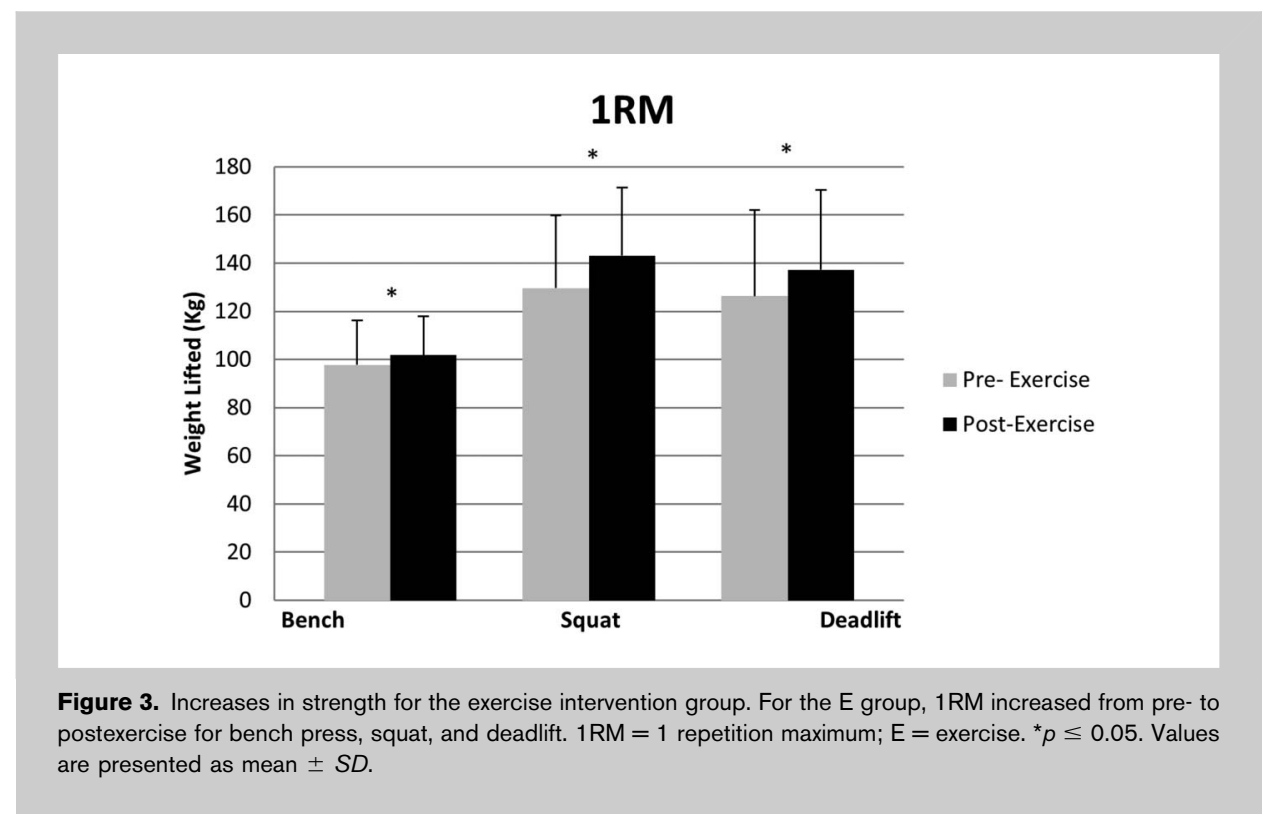

whereas the average fold change in plasma adiponectin expression in $\mathrm{C}$ group was $0.19 \pm 0.19$ (Figure 1). When the E group was separated into OD and ND subjects, changes in adiponectin were significantly different between the 2 populations $\left(p=0.046, \eta^{2}=0.034\right)$. The average fold change in plasma adiponectin expression in the OD subjects was $0.69 \pm 0.35$, whereas ND subjects exhibited almost no change in adiponectin $(-0.03 \pm 0.30$, Figure 2$)$. Thus, the differences in fold changes in plasma adiponectin found in the $\mathrm{E}$ group pre- to postexercise were primarily because of changes found in the OD subjects. The average fold change in plasma adiponectin expression in the OD subjects of the control group $(\mathrm{ODC})$ was $-0.47 \pm 0.07$, whereas the average fold change in plasma adiponectin expression in the ND subjects of the control group (NDC) was $0.35 \pm 0.57$, although differences were not significant. Similarly, fold changes in plasma adiponectin were not significantly different between OD subjects in the exercise group (ODE) vs. ODC because of the small number of OD subjects in each group, and no statistical differences were found between ND subjects in the exercise group (NDE) vs. NDC. Differences in fold changes between groups are summarized in Table 3.

Although not statistically significant, body fat percentage decreased from $19.3 \pm 1.3 \%$ pretraining to $17.5 \pm 1.1 \%$ posttraining in the $\mathrm{E}$ group, whereas no change occurred in the $\mathrm{C}$ group (23.8 $\pm 6.0 \%$ pretraining to $23.1 \pm 4.4 \%$ posttraining). Offspring of type 2 diabetic subjects in the control group had an initial body fat percentage of $22.2 \pm 6.25 \%$, whereas the NDC had an initial body fat percentage of $22.1 \pm 4.33 \%$. Similarly, ODE had an initial body fat percentage of $15.0 \pm$ $1.8 \%$, whereas the NDE had an initial body fat percentage of $19.4 \pm 4.9 \%$. The differences between OD and ND subjects were not significant for the $\mathrm{E}$ or $\mathrm{C}$ group at baseline. Changes in body composition for the NDC, ODC, ODE, and NDE are reported in Table 4. Differences between ODE and NDE groups were significant $(p<$ $\left.0.01, \eta^{2}=0.03\right)$. Neither differences between ODE and ODC groups were significant nor were any other between-group differences found for body fat percentage.

Body weight did not change significantly in either group or between OD and ND subjects. Calculated fatfree body mass (obtained by subtracting adipose tissue mass from body weight) increased in the $\mathrm{E}$ group from $64.8 \pm 6.1 \mathrm{~kg}$ before training to $67.8 \pm 7.0 \mathrm{~kg}$ after training but was not statistically significant. 
Regular aerobic exercise, changes in body fat percentage, and changes in body weight significantly correlated with changes in plasma adiponectin as shown in Table 5. Similarly, first-degree family history of type 2 diabetes significantly correlated with the fold change in adiponectin pre- to postintervention. Changes in plasma adiponectin concentration pre- to postintervention in the $\mathrm{E}$ group were no longer significant compared with the $\mathrm{C}$ group when adjusted for change in body weight, change in body fat percentage, or first-degree family history of type 2 diabetes.

As expected, strength, as measured by $1 \mathrm{RM}$, increased significantly in the E group after training as indicated in Figure 3. Bench press increased from $97.7 \pm 18.2$ to $101.8 \pm$ $16.4 \mathrm{~kg}$, an $8.2 \%$ increase, power $=0.80$. Squats increased from $129.5 \pm 30.5$ to $143.2 \pm 28.2 \mathrm{~kg}$, a $13.4 \%$ increase, power $=0.97$. Deadlifts increased from $126.4 \pm 35.5$ to $137.3 \pm 33.2 \mathrm{~kg}$, a $12.2 \%$ increase, power $=0.81$.

There were no significant changes in strength in the $\mathrm{C}$ group from pre- to postexercise intervention. Bench press decreased from $105.8 \pm 18.6$ to $104.9 \pm 21.4 \mathrm{~kg}$, squats decreased from $131.5 \pm 27.1$ to $126.9 \pm 29.1 \mathrm{~kg}$, and deadlifts increased from $131.8 \pm 27.0$ to $132.8 \pm 28.4 \mathrm{~kg}$.

\section{Discussion}

The present study suggests that a 12-week periodized highintensity resistance training program elicits minor improvements in circulating adiponectin levels, particularly among type 2 diabetic offspring. Furthermore, regular aerobic exercise and decreases in body weight or body fat percentage are associated with greater increases in plasma adiponectin. These findings are in agreement with previous studies that have shown increases in adiponectin after weight loss (37) or exercise in conjunction with weight loss (26). However, the present study did not demonstrate significant changes in body weight or body fat percentage for the exercise intervention group as a whole despite the significant increase in plasma adiponectin. Some studies have observed increases in adiponectin in the absence of weight loss among overweight or obese older populations $(22,31)$, but this is the first to identify changes among healthy populations. Previous long-term exercise training studies in healthy populations have, in large part, found no change $(17,29)$ and in some cases a decrease (18) in plasma adiponectin after training.

With the exception of the OD subjects in the exercise intervention group, the findings of the current study further support the notion that exercise interventions, in the absence of weight loss, are ineffective in affecting plasma adiponectin levels in healthy populations. There are several potential explanations as to why plasma adiponectin significantly increased among OD subjects in the exercise intervention group of the current study. One reason may be partially because of the high-intensity periodized resistance training protocol used. Fatouros et al. (13) determined that changes in adiponectin after resistance training are directly related to exercise intensity. Although exercise intensity varied from week to week in the current study, the overall program was considered "high intensity" for untrained individuals given that it elicited significant gains in strength as demonstrated by increases in 1RM for bench press, squats, and deadlifts. The majority of previous resistance training studies investigating adiponectin involved nonperiodized programs $(13,18,31)$. This type of program is less effective than periodized training programs for increasing strength (24), and therefore, metabolic, inflammatory, and endocrine adaptations are likely to be blunted as well. Given the enhanced gains in strength after a periodized training program compared with a nonperiodized training program, the current study suggests that adiponectin responses may also be enhanced. A recent study examining changes in plasma adiponectin and plasma leptin after 8 weeks of undulating periodization compared with nonperiodized or a linear periodization program noted a trend for greater strength gains, increases in adiponectin, and decreases in leptin (2). Though differences between exercise groups were not significant, the authors suggested that longer duration population-specific studies may reveal significant differences, as found in the current study. This further reinforces the notion that undulating periodization programs may enhance metabolic parameters more effectively than nonperiodized programs and therefore elicit superior health benefits in atrisk populations.

Although the type of training may have had a large effect on plasma adiponectin in the $\mathrm{E}$ group as a whole, a large degree of variation was found for individual responses to the exercise training. Some of this variation is likely because of genetic predisposition as seen in OD vs. ND subjects. As demonstrated in the current study and in previous studies, plasma adiponectin levels are highly variable among healthy $(14,17)$ and overweight, diabetic, or insulin-resistant populations $(34,35)$. Thus, changes in plasma adiponectin concentration pre- to postexercise intervention in OD and ND had considerable variation. Although most subjects in the E group increased circulating adiponectin pre- to postexercise, some actually decreased adiponectin pre- to postexercise. Some studies have shown decreases in adiponectin after exercise $(18,20)$, and it has been suggested that very high adiponectin levels are associated with all-cause mortality (8). Therefore, although absolute adiponectin concentration was not measured in the current study, it is possible that ND had higher initial adiponectin values at the onset of the study compared with OD, and as a result, decreases or no changes were observed in this population. This could be a protective effect to prevent too high of a concentration of this hormone. However, additional research is needed to investigate this issue in detail.

Data from the current study also demonstrated a greater decrease in body fat percentage in OD subjects compared with ND subjects in the E group. Although group differences were statistically significant, the number of ODE was only 3 , making the effect size relatively small. Furthermore, there is an estimated 3.5\% degree of error in a repeated 7 -site skinfold 
measurement (12). Although the same technician performed all skinfold measurements for all subjects pre- and postintervention, with such a limited sample size, it is difficult to attribute changes in body fat, which has a large degree of variability, directly to changes in adiponectin in the current study. Table 5 indicates that changes in body fat percentage had an inverse correlation with fold changes in adiponectin. A relationship between these 2 variables clearly exists and may have contributed to differences between groups, but no definitive statement can be made based on the data available in the current study. Thus, although weight loss and decreases in body fat percentage are linked with increased levels of adiponectin, other factors, such as overall inflammatory state (34) and reaching "optimal" levels of circulating adiponectin (8), may be more directly involved in the regulation of plasma adiponectin levels after long-term exercise training.

Given that weight loss is associated with elevated levels of adiponectin and decreased inflammatory markers (26), it is possible that the overall inflammatory state and potentially specific inflammatory signaling molecules account for some of the variations in plasma adiponectin expression in the current study and in previous studies. Even in the absence of weight loss, exercise has been shown to reduce inflammation in some cases (4), but not others $(29,30)$. The differences in these findings seem to be related to exercise intensity. Long-term high-intensity exercise yields a reduction in inflammation (3), whereas lower intensity exercise does not change inflammatory markers, including adiponectin (26). Therefore, although inflammatory markers were not measured in the current study, it is possible that the highintensity periodized resistance training program used in the current study induced changes in plasma adiponectin via a decrease in the overall inflammatory state of the individual.

Although it would have been ideal to have a greater number of OD subjects in both groups, we were limited in our population availability. Since our subject population average age was approximately 22 years, finding participants with type 2 diabetic parents proved to be a difficult undertaking. The incidence of type 2 diabetes is more prevalent in older individuals (36), and in large part, the average age of the participants' parents for the current study was less than 50 years, thereby limiting the OD population as a whole. Because of time constraints, we could not extend the study for an additional year to recruit additional participants, and thus, the OD vs. ND population was unbalanced. Furthermore, we recognize that the small number of OD participants is a limitation of current study, and further investigation is clearly warranted to more definitely establish differences in changes in plasma adiponectin expression after long-term exercise.

It is also worth noting that the relative level of plasma adiponectin for the current study was measured using a Western blot technique, as opposed to the measurement of absolute concentration of adiponectin via enzyme-linked immunosorbent assay or radioimmunoassay. Although specific concentration of adiponectin could not be determined pre- and postexercise using a Western blot technique, it did eliminate issues associated with plasma volume shifts (10). Also, plasma adiponectin concentration in humans varies considerably in healthy populations. Some studies report concentrations as low as $6 \mu \mathrm{g} / \mathrm{ml}(17)$, whereas other studies report concentrations as high as $20 \mu \mathrm{g} / \mathrm{ml}$ (14). Thus, the current study eliminated the issue of potentially skewed data by determining relative change rather than absolute values.

\section{Practical Applications}

In addition to the well-established neuromuscular and metabolic adaptations of periodized strength and conditioning programs, these programs may offer protective benefits in the prevention of type 2 diabetes via increased circulating adiponectin, particularly among OD who are at a greater disposition for developing the disease. Even among ND, periodized resistance training programs may help keep adiponectin levels in an optimal range that is associated with insulin sensitivity without reaching excessive levels that can be associated with detrimental health effects. The current data suggest that periodized resistance training is beneficial not only to athletes from a performance perspective but also to the general population from a health perspective. Furthermore, the current study and the study by Ahmadizad et al. (2) suggest that undulating periodization resistance training programs may offer superior metabolic benefits compared with linear or nonperiodized resistance training programs. Finally, the addition of regular aerobic exercise and weight loss can enhance the levels circulating adiponectin and further improve metabolic health in both ND and OD populations. Incorporating aerobic exercise into a periodized resistance training program, therefore, is likely to provide additional benefits. Further improvements may be seen in the case of weight loss or body fat loss.

\section{ACKnowledgments}

The authors have no conflicts of interest to declare. The authors would like to thank the Stephens laboratory personnel for their technical assistance with the Western blotting procedures. The results of the present study do not constitute endorsement of any products by the authors or the National Strength and Conditioning Association (NSCA). Funding for this study was provided by the Adipocyte Biology Laboratory at Pennington Biomedical Research Center.

\section{REFERENCES}

1. ACSM's Guidelines for Exercise Testing and Prescription. Baltimore, MD: Lippincott Williams \& Wilkins, 2013.

2. Ahmadizad, S, Ghorbani, S, Ghasemikaram, M, and Bahmanzadeh, M. Effects of short-term nonperiodized, linear periodized and daily undulating periodized resistance training on plasma adiponectin, leptin and insulin resistance. Clin Biochem 47: 417-422, 2014.

3. Arner, P, Arner, E, Hammarstedt, A, and Smith, U. Genetic predisposition for type 2 diabetes, but not for overweight/obesity, is associated with a restricted adipogenesis. PLoS One 6: e18284, 2011. 
4. Balducci, S, Zanuso, S, Nicolucci, A, Fernando, F, Cavallo, S, Cardelli, P, Fallucca, S, Alessi, E, Letizia, C, Jimenez, A, Fallucca, F, and Pugliese, G. Anti-inflammatory effect of exercise training in subjects with type 2 diabetes and the metabolic syndrome is dependent on exercise modalities and independent of weight loss. Nutr Metab Cardiovasc Dis 20: 608-617, 2010.

5. Blüher, M, Bullen, JW, Lee, JH, Kralisch, S, Fasshauer, M, Klöting, N, Niebauer, J, Schön, MR, Williams, CJ, and Mantzoros, CS. Circulating adiponectin and expression of adiponectin receptors in human skeletal muscle: Associations with metabolic parameters and insulin resistance and regulation by physical training. J Clin Endocrinol Metab 91: 2310-2316, 2006.

6. Bruce, CR, Kriketos, AD, Cooney, GJ, and Hawley, JA. Disassociation of muscle triglyceride content and insulin sensitivity after exercise training in patients with type 2 diabetes. Diabetologia 47: 23-30, 2004.

7. Church, TS, Blair, SN, Cocreham, S, Johannsen, N, Johnson, W, Kramer, K, Mikus, CR, Myers, V, Nauta, M, Rodarte, RQ, Sparks, L, Thompson, A, and Earnest, C. Effects of aerobic exercise training on hemoglobin A1c levels in patients with type 2 diabetes. JAMA 304: 2253-2262, 2010.

8. Dekker, JM, Funahashi, T, Nijpels, G, Pilz, S, Stehouwer, CDA, Snijder, MB, Bouter, LM, Matsuzawa, Y, Shimomura, I, and Heine, RJ. Prognostic value of adiponectin for cardiovascular disease and mortality. J Clin Endocrinol Metab 93: 1489-1496, 2008.

9. Diez, JJ and Iglesias, P. The role of the novel adipocyte-derived hormone adiponectin in human disease. Eur J Endocrinol 148: 293300, 2003.

10. Dill, DB and Costill, DL. Calculation of percentage changes in volumes of blood, plasma, and red cells in dehydration. $J$ Appl Physiol 37: 247-248, 1974.

11. Dunstan, DW, Daly, RM, Owen, N, Jolley, D, de Courten, M, Shaw, J, and Zimmet, P. High-intensity resistance training improves glycemic control in older patients with type 2 diabetes. Diabetes Care 25: 1729-1736, 2002

12. Durnin, JVGA and Rahaman, MM. The assessment of the amount of fat in the human body from measurements of skinfold thickness. Br J Nutr 21: 681-689, 1967.

13. Fatouros, IG, Tournis, S, Leontsini, D, Jamurtas, AZ, Sxina, M, Thomakos, P, Manousaki, M, Douroudos, I, Taxildaris, K, and Mitrakou, A. Leptin and adiponectin responses in overweight inactive elderly following resistance training and detraining are intensity related. J Clin Endocrinol Metab 90: 5970-5977, 2005.

14. Ferguson, MA, White, LJ, McCoy, S, Kim, H, Petty, T, and Wilsey, J Plasma adiponectin response to acute exercise in healthy subjects. Eur J Appl Physiol 91: 324-329, 2004.

15. Handschin, C and Spiegelman, BM. The role of exercise and PGC 1alpha in inflammation and chronic disease. Nature 454: 463-469, 2008.

16. Holten, MK, Zacho, M, Gaster, M, Juel, C, Wojtaszewski, JFP, and Dela, F. Strength training increases insulin-mediated glucose uptake, GLUT4 content, and insulin signaling in skeletal muscle in patients with type 2 diabetes. Diabetes 53: 294-305, 2004.

17. Hulver, MW, Zhang, D, Tanner, CJ, Houmard, JA, Kraus, WE, Slentz, CA, Sinha, MK, Pories, WJ, Macdonald, KG, and Dohm, GL. Adiponectin is not altered with exercise training despite enhanced insulin action. Am J Physiol Endocrinol Metab 283: E861-E865, 2002.

18. Ibáñez, J, Izquierdo, M, Martínez-Labari, C, Ortega, F, Grijalba, A, Forga, L, Idoate, F, García-Unciti, M, Fernández-Real, JM, and Gorostiaga, EM. Resistance training improves cardiovascular risk factors in obese women despite a significative decrease in serum adiponectin levels. Obesity (Silver Spring) 18: 535-541, 2010.

19. Jansson, PA, Pellme, F, Hammarstedt, A, Sandqvist, M, Brekke, H, Caidahl, K, Forsberg, M, Volkmann, R, Carvalho, E, Funahashi, T, Matsuzawa, Y, Wiklund, O, Yang, X, Taskinen, MR, and Smith, U. A novel cellular marker of insulin resistance and early atherosclerosis in humans is related to impaired fat cell differentiation and low adiponectin. FASEB J 17: 1434-1440, 2003.
20. Jürimäe, J, Purge, $P$, and Jürimäe, T. Adiponectin is altered after maximal exercise in highly trained male rowers. Eur J Appl Physiol 93: 502-505, 2005

21. Kelley, KR, Blaszczak, A, Haus, JM, Patrick-Melin, A, Fealy, CE, Solomon, TPJ, Kalinski, MI, and Kirwan, JP. A 7-d exercise program increases high-molecular weight adiponectin in obese adults. Med Sci Sports Exerc 44: 69-74, 2012.

22. Kirketos, AD, Gan, SK, Poynten, AP, Furler, SM, Chisholm, DJ, and Campbell, LV. Exercise increases adiponectin levels and insulin sensitivity in humans. Diabetes Care 27: 629-630, 2004.

23. Kramer, JB, Stone, MH, O’Bryant, HS, Conley, MS, Johnson, RL, Nieman, DC, Honeycutt, DR, and Hoke, TP. Effects of single vs multiple sets of weight training: Impact of volume, intensity, and variation. J Strength Cond Res 11: 143-147, 1997.

24. Kramer, WJ and Ratamess, NA. Fundamentals of resistance training: Progression and exercise perscription. Med Sci Sports Exerc 36: 674688, 2004.

25. Leaf, D, Parker, D, and Schadd, D. Changes in VO2max, physical activity, and body fat with chronic exercise: Effects on plasma lipid. Med Sci Sports Exerc 29: 1152-1159, 1997.

26. Madsen, EL, Rissanen, A, Bruun, JM, Skogstrand, K, Tonstad, S, Hougaard, DM, and Richelsen, B. Weight loss larger than $10 \%$ is needed for general improvement of levels of circulating adiponectin and markers of inflammation in obese subjects: A 3-year weight loss study. Eur J Endocrinol 158: 179-187, 2008.

27. Marcell, TJ, McAuley, KA, Traustadottir, T, and Reaven, PD. Exercise training is not associated with improved levels of C-reactive protein or adiponectin. Metabolism 54: 533-541, 2005.

28. Monteiro, A, Aoki, M, Evangelista, A, Alveno, D, Monteiro, G, Picarro, I, and Ugrinowitsch, C. Nonlinear periodization maximizes strength gains in split resistance training routines. $J$ Strength Cond Res 23: 1321-1326, 2009.

29. Nassis, GP, Papantakou, K, Skenderi, K, Triandafillopoulou, M, Kavouras, SA, Yannakoulia, M, Chrousos, GP, and Sidossis, LS. Aerobic exercise training improves insulin sensitivity without changes in body weight, body fat, adiponectin, and inflammatory markers in overweight and obese girls. Metabolism 54: 1472-1479, 2005.

30. Nikseresht, M, Agha-Alinejad, H, Azarbayjani, M, and Ebrahim, K. Effects of nonlinear resistance and aerobic interval training on cytokines and insulin resistance in sedentary men who are obese. $J$ Strength Cond Res 28: 2560-2568, 2014.

31. Olson, TP, Dengel, DR, Leon, AS, and Schmitz, KH. Changes in inflammatory biomarkers following one-year of moderate resistance training in overweight women. Int J Obes (Lond) 31: 996-1003, 2007.

32. Ouchi, $\mathrm{N}$ and Walsh, $\mathrm{K}$. Adiponectin as an anti-inflammatory factor. Clin Chim Acta 380: 24-30, 2007.

33. Prestes, J, Shiguemoto, G, Botero, JP, Frollini, A, Dias, R, Leite, R, Pereira, G, Magosso, R, Baldissera, V, Cavaglieri, C, and Perez, S. Effects of resistance training on resistin, leptin, cytokines, and muscle force in elderly post-menopausal women. J Sports Sci 27: 1607-1615, 2009.

34. Schulze, MB, Rimm, EB, Shai, I, Rifai, N, and Hu, FB. Relationship between adiponectin and glycemic control, blood lipids, and inflammatory markers in men with type 2 diabetes. Diabetes Care 27: 1680-1687, 2004.

35. Shand, BI, Scott, RS, Elder, PA, and George, PM. Plasma adiponectin in overweight, nondiabetic individuals with or without insulin resistance. Diabetes Obes Metab 5: 349-353, 2003.

36. Stratton, IM, Adler, AI, Neil, HAW, Matthews, DR, Manley, SE, Cull, CA, Hadden, D, Turner, RC, and Holman, RR. Association of glycaemia with macrovascular and microvascular complications of type 2 diabetes (UKPDS 35): Prospective observational study. BMJ 321: 405-412, 2000.

37. Yang, W, Lee, W, Funahashi, T, Tanaka, S, Yuji Matsuzawa, Y, Chao, C, Chen, C, Tai, T, and Chuang, L. Weight reduction increases plasma levels of an adipose-derived anti-inflammatory protein, adiponectin. J Clin Endocrinol Metab 86: 3815-3819, 2001. 